\title{
Integration of multimodal neuroimaging methods: a rationale for clinical applications of simultaneous EEG-fMRI
}

\author{
Paolo Vitali, MDa \\ Carol Di Perri, MDa \\ Anna Elisabetta Vaudano, MD, PhD \\ Stefano Meletti, MD, PhD ${ }^{\mathrm{b}}$ \\ Flavio Villani, MDc
}

a Brain MRI 3T Mondino Research Center, C. Mondino National Neurological Institute, Pavia, Italy ${ }^{\mathrm{b}}$ Department of Biomedical, Metabolic and Neural Science, University of Modena and Reggio Emilia, NOCSE Hospital, Modena, Italy

${ }^{c}$ Division of Clinical Epileptology \& Experimental Neurophysiology, IRCCS C. Besta Neurological Institute Foundation, Milan, Italy

Correspondence to: Paolo Vitali

E-mail: paolo.vitali@mondino.it

\section{Summary}

Functional magnetic resonance imaging (fMRI), which has high spatial resolution, is increasingly used to evaluate cerebral functions in neurological and psychiatric diseases. The main limitation of fMRI is that it detects neural activity indirectly, through the associated slow hemodynamic variations. Because neurovascular coupling can be regionally altered by pathological conditions or drugs, fMRI responses may not truly reflect neural activity. Electroencephalography (EEG) recordings, which directly detect neural activity with optimal temporal resolution, can now be obtained during fMRI data acquisition. Therefore, there is a growing interest in combining the techniques to obtain simultaneous EEG-fMRI recordings.

The EEG-fMRI approach has several promising clinical applications. The first is the detection of cortical areas involved in interictal and ictal epileptic activity. Second, combining evoked potentials with fMRI could be an accurate way to study eloquent cortical areas for the planning of neurosurgery or rehabilitation, circumventing the above-mentioned limitation of fMRI. Finally, the use of this approach to evaluate the functional connectivity of resting-state networks would extend the applications of EEG-fMRI to uncooperative or unconscious patients.
KEY WORDS: BOLD, epilepsy, neurovascular coupling, pre-surgical mapping, resting-state networks

Current clinical applications of fMRI and their limitations

Over the past two decades, functional neuroimaging has dramatically increased understanding of human brain functions. In earlier times, brain functions could be studied only indirectly, via neuropsychological tests administered to patients with brain lesions. Since the advent of computed tomography (CT) in the early seventies, brain lesions have been demonstrated in vivo using structural neuroimaging. Today, functional neuroimaging methods allow mostly non-invasive in vivo visualization of cerebral activity. However, to infer brain functions using these methods, it is important to recognize their limitations. The main limitation is that the method with the best spatial accuracy, i.e. blood oxygen level-dependent functional magnetic resonance imaging (BOLD fMRI), is based on hemodynamic changes in active areas, and therefore able to provide only indirect information on neural activity, based on the phenomenon of "neurovascular coupling", i.e. the relationship that exists between neural activity at cellular (neuronal and glial) and subcellular (neurotransmitters, chemical and electrical channels) levels and cerebral blood flow changes. Understanding neurovascular coupling is crucial in order to use this technique correctly in human diseases, in which this relationship is likely to be abnormal. In fact, although functional neuroimaging is currently used for research purposes in group studies, several specific issues (and many future expectations) need to be taken into account regarding the clinical application of functional neuroimaging at single-subject level.

In neurosurgery, fMRI is increasingly used in clinical routine to plan neurosurgical interventions. The aim in this setting is to achieve "safe" resection, i.e. to remove lesions without causing additional deficits. This is often possible for low-grade tumors or cortical dysplasias in a functional area (i.e. motor or language). In such cases of congenital or slowly-growing lesions, functions progressively reorganize in the adjacent areas. Imaging this functional reorganization, driven by brain plasticity, is crucial for surgical planning, as it has been demonstrated that a lesion-to-activation distance of less than $5 \mathrm{~mm}$ is correlated with additional post-surgical deficits (Krishnan et al., 2004). 
In the case of patients with low-grade tumors (Benzagmout et al., 2007) or with cortical dysplasias (Vitali et al., 2008), studying brain reorganization may allow a larger resection and a better outcome without any functional deficit. In temporal lobe epilepsy, the functional shift of language and memory areas to the non-dominant hemisphere correlates with minimal post-surgical deficits, or even improvements, in naming and verbal memory performance (Rosazza et al., 2013). However, there is an important issue to be considered: in tumors and vascular malformations, neurovascular coupling is often impaired due to pathological vascular proliferation (Fujiwara et al., 2004). To cross-validate fMRI results, each fMRI activation is usually checked by intraoperative electrocortical stimulation during awake surgery. However, awake surgery is time consuming and may be stressful for the patient. It would, therefore, be more efficient to combine $\mathrm{fMRI}$ maps with neurophysiological data not based on neurovascular coupling - data obtained before surgery using techniques such as evoked potentials (EPs), magnetoencephalography (MEG) or transcranial magnetic stimulation (TMS).

In neurology, there could, in the near future, be a role for functional neuroimaging at single-subject level; for instance, when planning rehabilitation treatments after stroke. In fact, functional neuroimaging studies are increasingly showing that the activation pattern can identify patients more likely to improve in the affected function and, therefore, help to establish where to focus rehabilitation efforts (Gale and Pearson, 2012). However, in cerebrovascular patients, the actual BOLD signal may underestimate the cortical activity near the ischemic area, as demonstrated by studies combining fMRI with neurophysiological techniques. For instance in one study, motor activations after stroke not detected by $f M R I$ were readily detected by MEG in patients with impaired cerebrovascular reactivity, as assessed by transcranial Doppler (Rossini et al., 2004).

Another emerging field of clinical application for fMRI is the evaluation of residual cerebral functions in patients with consciousness disorders such as the vegetative state (VS) or minimally conscious state (MCS). Neurovascular coupling is also likely to be affected in these patients presenting severe postanoxic or post-traumatic encephalopathy. Moreover, although fMRI can detect wilful modulation of brain activity in some patients without signs of awareness (Monti et al., 2010), it would be more feasible in the clinical setting to detect residual cerebral functions at the bedside using EEG/EPs (Cruse et al., 2012).

In the near future, fMRI is likely to become increasingly important in other medical fields: in cardiology, for instance, given that the heart and brain are reciprocally linked. In fact, epileptic seizures arising from the insula cause dysrhythmia and even cardiogenic syncope. The epileptogenic area in these patients could be evaluated by simultaneous EEG-fMRI recording, a method described in more detail further on. On the other hand, insula involvement has also been reported in patients with ischemic heart disease (Nagai et al., 2010). It is therefore likely that study of sponta- neous transitory events, such as epileptic seizures and syncope, will be coupled with electrophysiological monitoring during the $\mathrm{fMRI}$ session.

As reviewed here, in most of the clinical fields where fMRI currently plays an important role (or will do so in the future), combining it with electrophysiological recording is a highly desirable way of obtaining direct measurement of cortical activity, avoiding the need to rely on neurovascular coupling. However, combining these methods requires knowledge of the signal measured by each of them.

\section{Where does the BOLD signal arise from? Neurovascular coupling}

As the acronym indicates, the BOLD signal detected in $\mathrm{FMRI}$ is dependent on the level of blood oxygenation. The signal recorded from the scanner is $\mathrm{T}^{*}$, the transverse relaxation time (T2) plus the component related to the local variations in magnetic susceptibility due to the variations in deoxyhemoglobin $(\mathrm{Hb})$ concentration.

In 1992, two different groups simultaneously demonstrated a BOLD signal increase (up to $5 \%$ ) in human sensory areas during functional activation (Ogawa et al., 1992; Kwong et al., 1992). In fact, significant variations in $\mathrm{Hb}$ occur in capillaries and venules draining blood from activated functional areas. Arteriolar vasodilatation produces an increase in cerebral blood flow, with delivery of fresh arterial blood, which increases the oxyhemoglobin/deoxyhemoglobin $(\mathrm{HbO} / \mathrm{Hb})$ concentration ratio, overcompensating for the oxygen consumption. Near infrared spectroscopy studies allowed a more precise description of the $\mathrm{HbO} / \mathrm{Hb}$ time course: within the first second of neural activity there is a small (1\%) increase in $\mathrm{Hb}$ (and decrease in $\mathrm{HbO}$ and in the BOLD signal), followed by a larger decrease in $\mathrm{Hb}$ (and an increase in $\mathrm{HbO}$ and the BOLD signal) due to local vasodilatation, reaching a $5 \%$ peak within 4-6 seconds. With magnetic fields higher than 3 Tesla, the initial negative variation of the BOLD signal can be detected (Cannestra et al., 2001), but the poor signal-to-noise ratio at the current magnetic fields prevents it from being used for functional mapping. A recent $3 T$ fMRI study on the visual cortex (Watanabe et al., 2013), which showed a significant trial-to-trial variability for the positive peak, uncovered the initial dip in the majority of voxels by pooling trial responses with large peak latencies.

BOLD fMRI studies are currently carried out considering the 3-5\% BOLD increase 4-6 seconds after the stimulus. This latency between neural activity and BOLD signal is an intrinsic limitation of BOLD fMRI, further complicated by the inhomogeneity of this latency in different brain regions (Chang et al., 2008), which is particularly marked in diseases affecting cerebrovascular reactivity. Another intrinsic limitation of BOLD $\mathrm{FMRI}$ is that local variations of cerebral blood flow during activation have to be considered taking into account the global variation of basal cerebral blood flow in the resting state. 
Basal perfusion can be non-invasively quantified on MRI by arterial spin labeling, and recent studies have shown that dynamic changes in both blood flow and volume can be measured simultaneously by means of dedicated sequence implementation. Since changes in blood flow, compared with BOLD changes, have been demonstrated to be related more specifically to the cortical capillaries (Raoult et al., 2011), these methods may replace BOLD imaging when very high magnetic field scanners $(\geq 7 \mathrm{~T})$ are available for clinical purposes.

Using very high spatial resolution (isotropic voxel 1 $\mathrm{mm}^{3}$ ) spin-echo-based echo-planar imaging (EPI) at 9.4T, even the BOLD signal can be confined within the intracortical capillaries, and the effect of large veins can be minimized (Budde et al., 2014). Specificity and spatial resolution are the main advantages of a very high magnetic field. The third advantage, increased BOLD sensitivity at $7 \mathrm{~T}$, is already evident even with medium spatial resolution EPI (voxel size $2 \times 2 \times 3 \mathrm{~mm}^{3}$ ), as demonstrated in a presurgical setting (Beisteiner et al., 2011). However, before the 7T scanner can be used in clinical settings, safety issues have to be carefully addressed and technical issues overcome in order to achieve a uniform static magnetic field shim and a uniform radiofrequency excitation field.

Despite its intrinsic limitations, BOLD $\mathrm{fMRI}$ is the best available tool for brain mapping. To compensate for its poor temporal resolution, simultaneous acquisition of electrophysiological data would be a perfect integration. A number of studies have demonstrated that most of the EEG-recorded activity reflects the excitatory and inhibitory post-synaptic potentials.

While both methods, fMRI and EEG, can detect, in vivo, cerebral activity associated with sensory, motor or cognitive tasks, it still has to be demonstrated that the neural activity recorded by the two methods is exactly the same. To obtain the two signals from the exact same region, Logothetis et al. (2001) used micro-coils coaxial to intracerebral electrodes implanted in the monkey occipital cortex. Using this sophisticated approach, they demonstrated that visual stimulation causes an almost simultaneous increase in BOLD signal and electrophysiological activity. Then, after dividing the electrophysiological activity into high and low frequencies, they showed that the BOLD signal is associated more with low-frequency activity, i.e. the synaptic activity of the local field potentials (LFPs), than with high-frequency activity, i.e. the intracellular multi-unit spiking activity. Other studies in monkeys confirmed the BOLD-LFP correlation (Issa et al., 2013).

\section{Simultaneous EEG-fMRI recording}

Since the first "Human Brain Mapping" conference (Paris, 1994), there has been a growing trend to combine functional neuroimaging with electrophysiological methods. At that 1994 meeting, a pioneering study by the Boston group of Warach and colleagues showed that EEG signals can be recorded during fMRI (Ives et al., 1993). To date, several different commercial systems have been developed to allow this combination, which is technically difficult due to a number of novel EEG artifacts. Various methods have been developed to reduce these artifacts (Allen et al., 1998, 2000) and considerable efforts have been made to maximize the amplitude of potentially clinically-relevant information from single EEG-fMRI datasets (Grouiller et al., 2011; Storti et al., 2013; Ruggieri et al., 2015).

In cognitive neuroscience, simultaneous EEG-fMRI studies provide information that is additional to the data deriving from the merging of separate acquisitions. In fact, trial-to-trial variability of event-related potentials in the classical oddball task (Warbrick et al., 2009) or other cognitive tasks (Wirisch et al., 2014) may be employed as a regressor to improve the event-related fMRI analysis obtained by standard stimulus onset timing. This is especially relevant to pharmacological EEG-fMRI studies (Diukova et al., 2012), in which neurovascular coupling can be affected. In other EEG-fMRI studies using visual attention (Scheringa et al., 2011) or hand grip (Sclocco et al., 2014) tasks, EEG informed analysis was performed and confirmed a previously proposed "heuristic" model. This model (Kilner et al., 2005) proposed that BOLD activations are associated with an increase in the 'average' frequency of EEG, where average is defined as the root mean square, with a reduction in amplitude. Thus, increases in higher frequencies (gamma), relative to lower frequencies (alpha range), lead to BOLD signal increases. Interestingly, this model can also explain EEG-fMRI data in epileptic patients (Meletti et al., 2014).

Scalp EEG is currently carried out, for clinical purposes, using a limited number of electrodes, usually 21 , with 19 recording channels, according to the standard 10-20 international system. In the last few years, research laboratories using high-density recording systems (more than 64, up to 256 channels) and complex algorithms to identify signal source have improved the spatial resolution of the events recorded from the cerebral cortex. Therefore, high-density EEG can today be considered a neuroimaging method (Michel and Murray, 2012). In epilepsy in particular, electrical source imaging (ESI) allows the electrical sources underlying scalp potential maps to be estimated with a temporal resolution in the order of milliseconds. Several studies have now confirmed its role as an accurate tool for estimating the source of focal epilepsy (Michel and Murray, 2012). However, the spatial resolution obtained by scalp EEG is intrinsically limited, because the electrode is located at a distance of about $1 \mathrm{~cm}$ from the cortex, a space within which skin, skull, three layers of meninges, cerebrospinal fluid and vessels determine a significant low-pass filter of activity recorded on the scalp. Moreover, activity within deep brain structures such as the hippocampus, amygdala and basal ganglia is unlikely to be detected.

$E E G$, independently of the number of channels, allows online recording of cerebral activity with virtually real temporal resolution. This direct window onto the cere- 
bral processes is an unbeatable feature of EEG, intrinsically impossible using pure imaging tools.

For this reason, the combination of the high temporal resolution of EEG and the high spatial resolution of fMRI offers a powerful integration of the respective complementary strengths of both methods.

Simultaneous EEG-fMRI recording provides two different datasets: well localized hemodynamic variations only indirectly related to the neural activity, and poorly localized electrophysiological signals directly reflecting the neural activity. While the first EEG-fMRI recordings were spike-triggered acquisitions (Fig.1) the development of effective means of removing MRIgradient artifacts and pulse-related artifacts has made it possible to record EEG continuously and simultaneously during fMRI (Allen et al., 2000) (Fig.s 2,3), allowing much improved modeling of the BOLD signal. An interesting insight into the relationship between scalp $E E G$ and $\mathrm{AMRI}$ signal is provided by EEG-fMRI recordings performed in epileptic patients. Simultaneous EEG and $\mathrm{fMRI}$ can detect cerebral hemodynamic changes related to interictal epileptiform discharges (IEDs) and even seizures identified on scalp EEG (Chaudhary et al., 2013; Vaudano et al., 2014) (see as a paradigmatic example, Fig. 2). IEDs arise from the "irritative zone", which is partly or completely enclosed in the cortical region causing the seizures ("epileptogenic zone"; EZ). In patients with refractory focal epilepsy the significant clinical question is how the EEG-fMRI results might help to localize the EZ. To date, the intracranial EEG (icEEG) recording is considered the gold standard for identifying the EZ, although it is expensive and has associated morbidity. Recent EEG-fMRI studies on large populations of patients with focal epilepsy showed that the brain regions responsible for IED generation often closely matched the EZ (Thornton et al., 2010; Pittau et al., 2012a). More importantly, it has been demonstrated that when the surgical resection completely removes the region in which IEDs generate the BOLD signal change, a good outcome and seizure freedom are obtained (Thornton et al., 2010). Several studies have validated EEG-fMRI results versus the gold standard of icEEG localization of the EZ, and versus different parameters of surgical outcome (Thornton et al., 2010; Grouiller et al., 2011; Pittau et al., 2012a). Despite their differences, these papers showed that EEG-fMRI can provide useful additional information in the non-invasive pre-surgical evaluation (Fig. 3).

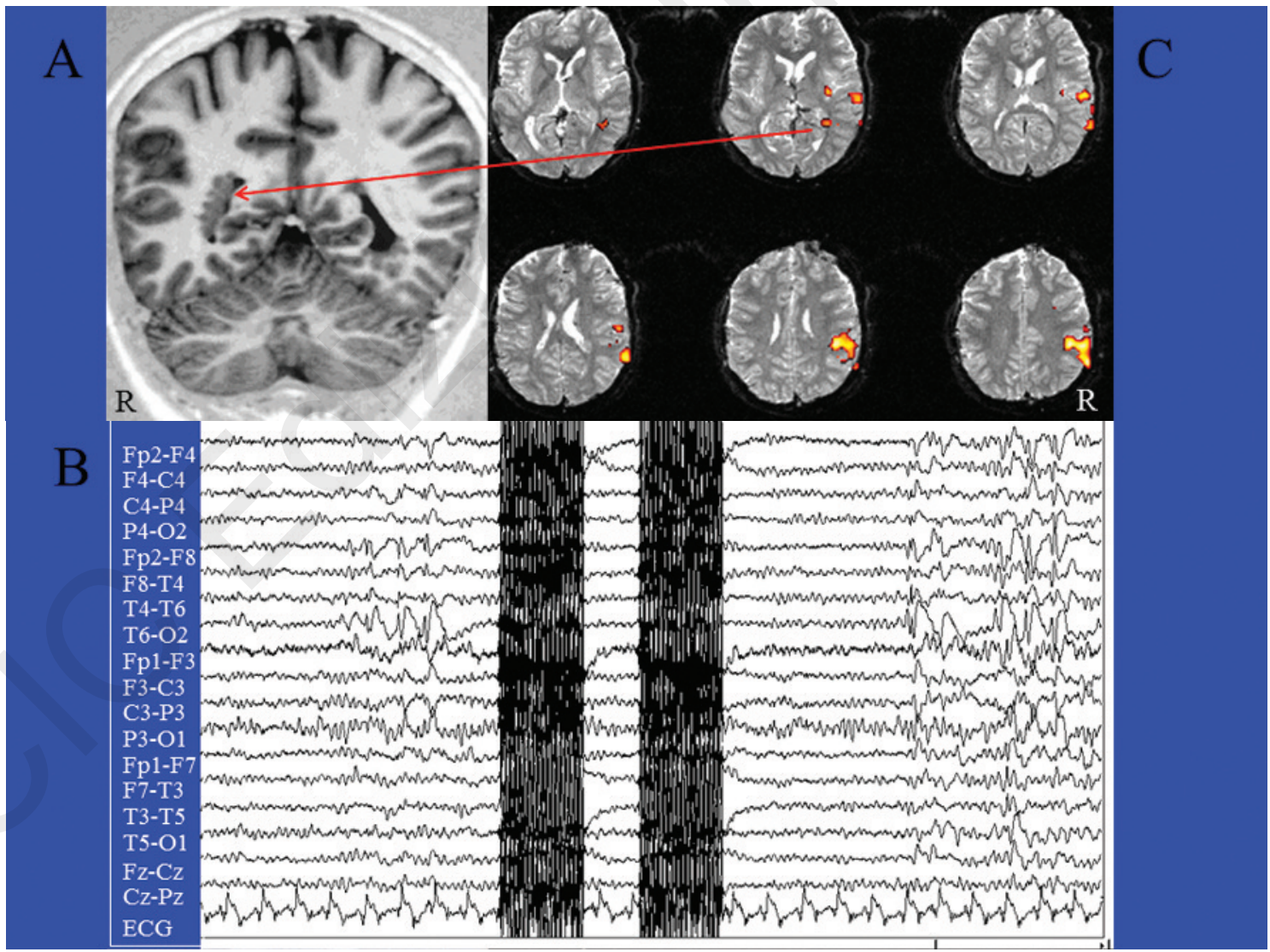

Figure 1 - The classical spike-triggered method in a case of focal epilepsy.

Patient (female, 23 yrs) with focal epilepsy and multiple nodules of subependymal heterotopia, mainly in the right hemisphere (A). EEG-fMRI acquisition of two EPI scans triggered by interictal epileptic activities (spikes and waves) recorded on the scalp, mainly from the T4-T6-P4 electrodes (B). After acquiring an equal number of EPI scans without spikes for at least 25 ", the interictal and basal images were statistically compared by t-test analysis (statistical parametric mapping). The fMRI map disclosed right temporoparietal areas of BOLD decrease, including a deep cluster located within one of the subependymal nodules (C). 
IED-related fMRI activations are usually identified by applying the general linear model (GLM), which employs the expected shape of the BOLD response due to stimulus presentation, i.e. hemodynamic response function (HRF). The convolution of timing of abnormal EEG events, represented as stick functions or blocks, with the expected HRF provides the regressor for the GLM analysis of fMRI data. Maps showing regions of significant IED-related changes are obtained through voxel-wise fitting of the model and application of appropriate statistical thresholds. The main limitation of the standard approach is that it uses, as a model of HRF, the method based on eventrelated $\mathrm{fMRI}$ data measurement during acoustic stimulation in the primary auditory cortex (Glover, 1999). This HRF model is unlikely to reflect the exact response in different brain areas in normal subjects, and even less likely to do so in epileptic patients (Benar et al., 2002). As pointed out by CaballeroGaudes et al. (2013), the variability in HRF has been addressed using different approaches: i) "incorporating additional basis functions such as the temporal derivative and the dispersion derivatives of the canonical HRF" (Salek-Haddadi et al., 2006); ii) "adopting a model for the HRF with no shape constraints such as the finite impulse response" (van Houdt et al., 2010); iii) "considering Fourier sets" (Lemieux et al., 2008); and iv) "introducing multiple HRF shapes with successive latencies" (Benuzzi et al., 2012). The degree of correlation between the EEG-fMRI results and the EEG focus increases when using a flexible approach in which HRF is estimated from the data (van Houdt and Zijlmans, 2013; Storti et al., 2013).

As opposed to model-based approaches, exploratory data-driven techniques of $\mathrm{FMRI}$ analysis, such as independent component analysis (ICA) (Thornton et al., 2010), or temporal clustering analysis (Morgan et al., 2008), have shown their ability to capture BOLD signal changes without imposing constraints on the HRF shape. To date, despite the mentioned evidence, there is no definite consensus on which HRF model and thresholds are the best options for localizing the generators of epileptic activity, and the clinical impact of this choice when patients are referred for surgery
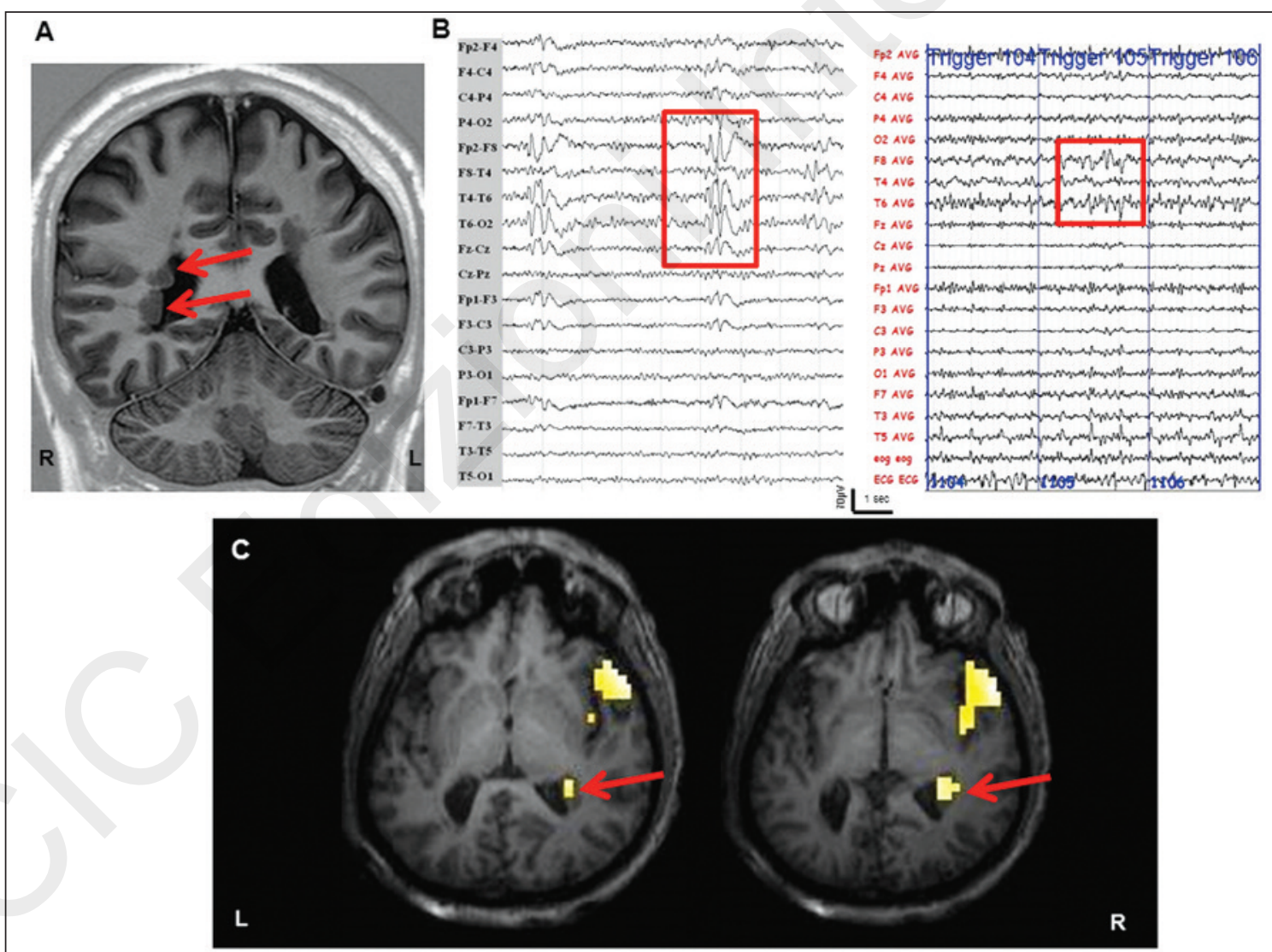

Figure 2 - Continuous EEG-fMRI in focal epilepsy.

Structural MRI scan shows bilateral periventricular nodular heterotopia (Panel A, red arrows). Scalp EEG demonstrates short sequences of interictal spike and spike-wave discharges mainly on the right frontotemporal leads, highlighted in the red box (Panel B, left image). Panel B, right image shows the EEG recorded simultaneously during fMRI acquisitions after off-line subtraction of gradient artifacts. The EEG trace (32 channels) is displayed in bipolar montage. The consecutive numbers on the EEG (in blue) indicate the corresponding EPI acquired. Note the presence of interictal abnormalities over the right frontotemporal regions, highlighted by the red box. Panel C: IED-related BOLD results ( $<0.05$ corrected for family-wise errors). SPM8 software (http://www.fil.ion.ucl.ac.uk/spm/) was used for data analysis. Main clusters of BOLD signal increase (global maxima) were detected at the right temporopolar cortex and at the right nodules of subependymal heterotopia. No decrease in BOLD signal was observed. The fMRI results are displayed on the patient's high-resolution T1-weighted structural scan. L: left; R: right. 
remains an open issue (van Houdt and Zijlmans, 2013). An innovative approach was recently proposed which involved neither a priori models of HRF shape, nor knowledge about the timing of the IED events (Caballero-Gaudes et al., 2013). This is a clear advantage, because accurate IED labeling is time consuming and requires experienced reviewers, while inaccurate IED recognition and marking has been shown to be an important source of error on the related hemodynamic maps. Recent approaches with quantitative automatic analysis of the intrascanner EEG led to increased sensitivity and specificity of fMRI maps, with faster processing (Pedreira et al., 2014).

Furthermore, several studies have proposed alternative or complementary strategies to improve EEG-guided $\mathrm{fMRI}$ analysis beyond spike labeling. Given the high proportion of patients without spikes during EEG-fMRI recordings, techniques based on correlation between the topographic features of spikes recorded on EEG outside the scanner with EEG recordings inside the scanner can allow epileptogenic focus detection in patients with otherwise inconclusive studies (Grouiller et al., 2011). Even in the presence of IEDs, the sensitivity of EEG-fMRI was improved by combining the conventional IED-related analysis with ESI of the same interictal events (Vulliemoz et al., 2010).

IED-related multifocal positive and negative hemodynamic changes have been found in areas distant from the presumed epileptic focus (Fahoum et al., 2012). These findings have raised questions about how hemodynamic responses are related to these events, given that neurovascular coupling could be altered in epilepsy. Moreover, cortical and subcortical BOLD responses have been detected prior to the EEG abnormalities revealed on scalp EEG, in both focal and generalized epilepsies (Moeller et al., 2008; Jacobs et al., 2009). While the significance of the early BOLD response is debated, these findings may depend on the poor sensitivity of scalp EEG, which does not reflect the abundant underlying epileptic

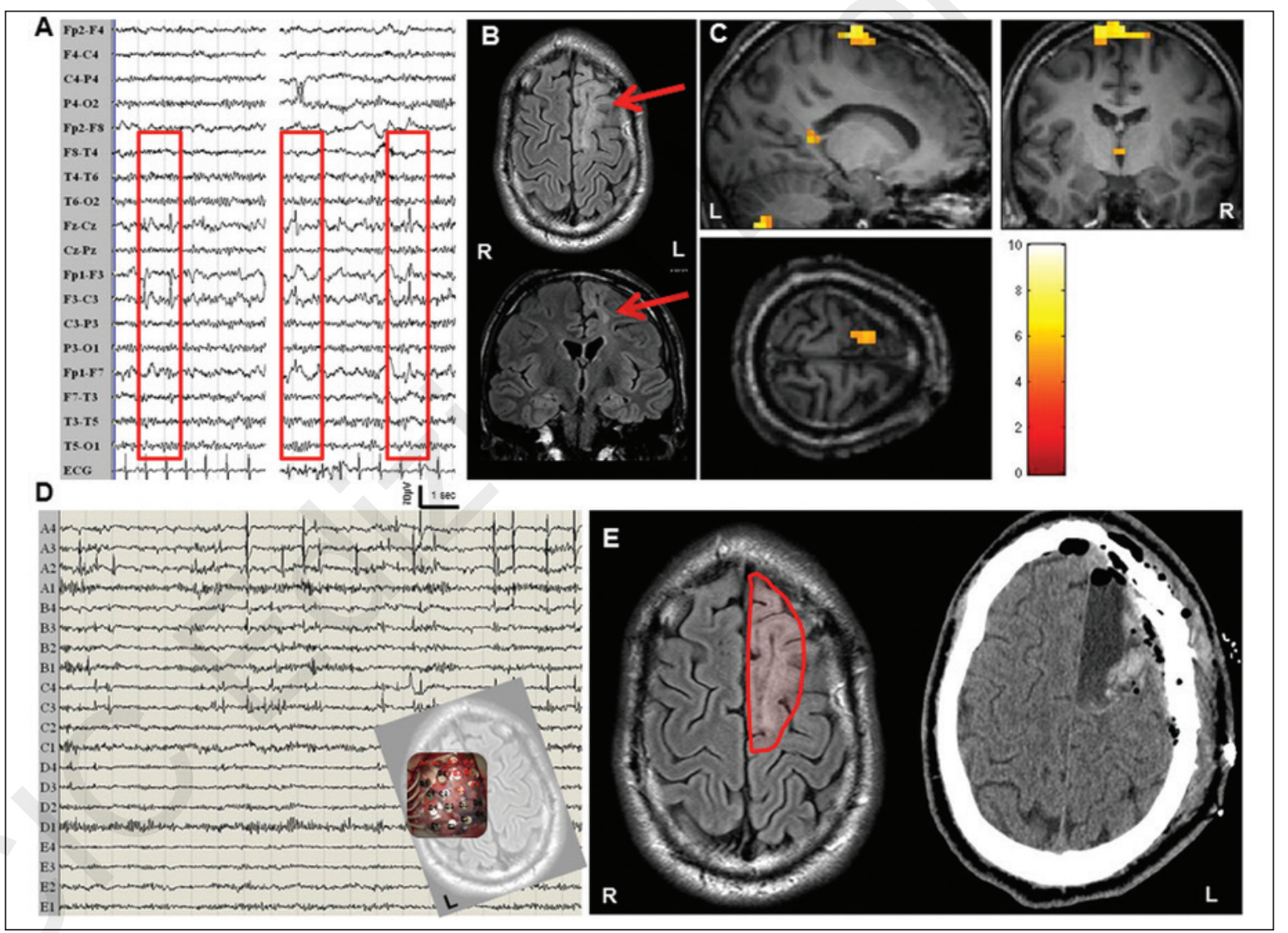

Figure 3 - Continuous EEG-fMRI. Another case of focal epilepsy, with iEEG and good post-operative outcome.

Interictal scalp EEG demonstrated isolated spikes and sharp waves over the frontocentral leads (Panel A). Panel B shows the patient's structural FLAIR images (coronal and axial slices): the red arrow indicates a large cortical gliotic lesion over the left frontomedial region. Panel C: IED-related BOLD results derived from simultaneous acquisition of EEG and fMRI data ( $\mathrm{p}<0.05$ corrected for family-wise errors). SPM8 software (http://www.fil.ion.ucl.ac.uk/spm/) was used for data analysis. A main cluster of BOLD signal increase (global maxima) was detected at the left frontomedial cortex, overlapping the lesion. Other blobs were detected at the ipsilateral cerebellum and thalamus and a small cluster was detected over the left posterior cingulate. No decreases in BOLD signal were detected. The fMRI results are displayed on the patient's high-resolution T1-weighted structural scan. Panel D: representative page of the intracranial recordings with subdural electrodes performed for pre-surgical purposes. A $5 \times 4$ grid was located over the left frontomedial cortex covering the structural lesion (small snapshot at the bottom right of the image). The electrodes A1, A2, A3, A4, B3, B4, C3, C4 (highlighted in red) showed continuous interictal spiking. Panel E: following the icEEG recording, a lesionectomy was performed (left image, the continuous red line indicates the boundary of the surgical resection). The right image shows a CT scan performed after surgery. The patient is seizure-free at three years of follow-up (ILAE, Class la). L: left; R: right. 
activity recorded by intracranial EEG (Tao et al., 2005). Answers to these questions might be found thanks to the recent possibility of recording intracranial EEG and fMRI simultaneously (icEEG-fMRI). With the support of careful safety studies even at $3 T$ (Boucousis et al., 2012), to date four patients with focal epilepsy have been investigated by icEEG-fMRI (Vulliemoz et al., 2011; Cunningham et al., 2012). These preliminary studies have demonstrated a good correspondence between the pathological activity revealed by icEEG and significant hemodynamic changes revealed by fMRI.

Because icEEG (whether or not it is combined with $f M R I)$ is intrinsically invasive, scalp EEG-fMRI remains a valid non-invasive tool for exploring epileptic networks, especially given the ongoing efforts to develop approaches to improve its sensitivity and specificity. Recent EEG-fMRI fusion strategies imply an analysis model that explicitly takes into account the "local coupling/uncoupling of bioelectric and hemodynamic activities, which are supposed to share a common substrate" (Daunizeau et al., 2007). To date, however, the application of such models in epilepsy is limited to single case documentation (Daunizeau et al., 2007).

As highlighted by Vaudano et al. (2013), an important limitation of scalp EEG-fMRI studies in clinical practice, and especially for surgeons, "resides in the interpretation of multiple clusters of BOLD signal changes: which one(s) represent(s) the site of IED/seizure ori- gin and which are involved due to propagation?". The temporal resolution of the BOLD signal limits the sensitivity of $\mathrm{fMRI}$ to identify the driver of the activity. Nevertheless, fMRI data analyses have recently been implemented to evaluate "effective connectivity", and this approach can be employed to assess the causal hierarchy within an epileptic network. Using an effective connectivity analysis (namely dynamic causal modeling), Vaudano et al. (2013) showed the applicability of this method, compared with icEEG, for identifying the EZ and the "propagation network". Similarly, other groups have shown that combining ESI and fMRI makes it possible to distinguish between hemodynamic changes related to IED onset versus propagation (Vulliemoz et al., 2010). Partial correlation analysis has also been applied to identify which of the irritative zones revealed by BOLD analyses was the first involved in the interictal activity, therefore playing a primary role in the epileptogenic network (Tana et al., 2008). Finally, new sequences are being developed to improve the temporal resolution of BOLD fMRI. A so-called magnetic-resonance-encephalography (MREG) sequence has recently been proposed to obtain temporal resolution of around $100 \mathrm{~ms}$. Preliminary application in 13 patients with focal epilepsy showed that the number of patients in whom the BOLD responses correlated with the spike topography was higher with MREG than with conventional EPI (Jacobs et al., 2014). Temporal and spatial resolution of BOLD fMRI will improve in the near future, especial-
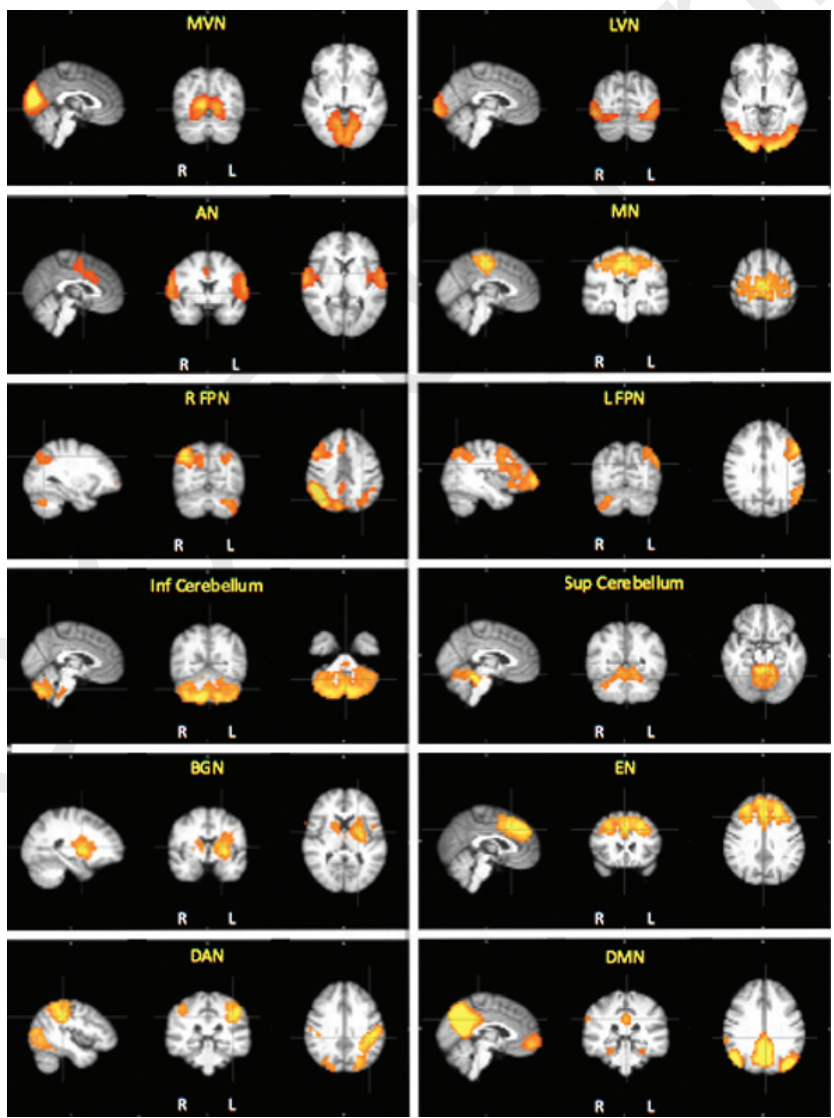

Figure 4 - The standard networks usually identified in rsfMRI.

Twelve resting-state networks identified by independent component analysis (by MELODIC, part of FSL software package) of the total fMRI data from 27 elderly subjects (mean age: 65 years).

$M V N$ and $L V N=$ medial visual and lateral visual networks; $A N=a u d i t o-$ ry network; $M N=$ =motor network; $R$ FPN and L FPN=right and left frontoparietal networks; Inf and Sup Cerebellum=inferior and superior

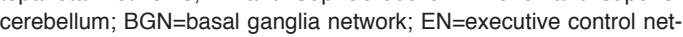
work: DAN=dorsal attentional network; $\mathrm{DMN}=$ default mode network. All images are $z$-statistics thresholded at $p<0.05$, overlaid onto the average scan in the standard MNI152 space, according to radiological convention. Figure courtesy of Dr Letizia Casiraghi. 
ly with very high magnetic fields. Preliminary safety studies on humans showed that EEG-fMRI is feasible even in a 7T scanner (Jorge et al., 2015).

\section{Future trends in functional neuroimaging research: the resting-state networks}

Outside the field of epilepsy, other simultaneous EEG$\mathrm{fMRI}$ studies have investigated the time course of $\mathrm{fMRI}$ activation using EEG simultaneously recorded in the same areas during sensory (Anwar et al., 2009), motor (Sclocco et al., 2014) or cognitive tasks (Warbrick et al., 2009; Scheringa et al., 2011; Wirisch et al., 2014). However, it is not necessary to use a stimulation task to explore functional activity in the brain. Resting-state networks (RSNs) are physiological neural networks detected by spontaneous oscillations of the BOLD signal that should be intrinsically linked to the spontaneous oscillations of the EEG signal.

Resting-state functional magnetic resonance imaging (rs-fMRI) is a non-invasive technique used to investigate the spontaneous temporal fluctuations in the BOLD signal. Physiological parameters, such as cardiac and respiratory rate, may contribute in part to these oscillations (Birn et al., 2012). These oscillations correlate within areas involved in the same functional circuit and the oscillations in that circuit survive after subtraction of the physiological variations. These facts suggest that these oscillations are probably related to neural networks, and are an expression of synchronized neural activity (i.e. functional connectivity) existing between different brain locations in a given period of time in the absence of input (sensory) or output (motor) tasks.

There are two main methodological approaches to study functional connectivity in RSNs by fMRI: one is a completely data-driven approach involving ICA, the other relies on prior anatomical hypotheses so as to restrict the analysis to a predefined set of seed regions of interest (ROIs) or to one specific seed ROI. When the analysis is performed by a set of predefined seed ROIs (i.e. anatomically defined), the two methods can be compared on the same dataset, although the results of the ICA still depend on the chosen number of independent components (Ma et al., 2007).

One study (Rosazza et al., 2012) showed that when selecting 20 independent components, there was a significant correspondence between the results obtained with the two approaches. The same group analyzed, at single-patient level, rs-fMRI in disorders of consciousness, and showed that the two approaches are differently sensitive to the steps of the processing pipeline (Andronache, 2013). They also showed that head motion, without appropriate multi-steps processing, tends to cause false negative correlations (underestimation) in ICA and false positive correlations (artifactual overestimation) in seed analysis. The two approaches can also disclose complementary results, as shown by another recent study, also in the field of disorders of consciousness (Di Perri et al., 2013). In uncooperative patients, the steps of the processing pipeline are particularly relevant because
fMRI, even rs-fMRI, has been proven to be very sensitive to head motion (Power et al., 2012).

While several RSNs with high spatial consistency across subjects have been detected using ICA (Beckmann and Smith, 2005), a single specific rs-fMRI network, referred to as the "default mode" network (DMN) (Raichle et al., 2001), has recently attracted great interest in the clinical neuroscience community. The DMN has been defined as a set of areas, encompassing the posterior cingulate/precuneus, anterior cingulate/mesiofrontal cortex, and temporoparietal junctions, which show more activity at rest than during attention-demanding tasks (Raichle et al., 2001). The significance of this network is still speculative, but a role in cognition, particularly memory, or consciousness has been proposed. So far, most of the work and interest concerning the RSN and the DMN has focused on dementia, especially Alzheimer's disease (AD).

Greicius et al. (2004) first showed that DMN activity in the precuneus/posterior cingulate cortex and hippocampus is lower in AD patients than in healthy elderly people, with a high sensitivity and specificity, and this finding has been replicated by several studies. Zhou et al. (2010) were able, with high accuracy, to distinguish $A D$ patients not only from controls but also from patients with frontotemporal dementia. Even normal elderly adult subjects at risk of developing $A D$, because of amyloid plaques on positron emission tomography, showed DMN disruptions (Sheline et al., 2010). Measures of functional connectivity within the DMN correlate with conversion from mild cognitive impairment to AD (Petrella et al., 2011), and combined with other MRI parameters are likely to be useful for diagnostic purposes (Dai et al., 2012).

Moreover, recent studies demonstrated that the functional connectivity derangement in $\mathrm{AD}$ extends beyond the DMN (Castellazzi et al., 2014) and in early phases of the disease is associated not only with decreased connectivity, but also with increased connectivity, especially in frontal regions (Damoiseaux et al., 2012; Castellazzi et al., 2014).

The links between the DMN and memory and executive functions in normal and pathological conditions may have profound implications for consciousness. fMRI studies have identified a clear reduction of DMN connectivity in coma, VS and MCS (Vanhaudenhuyse et al., 2010; Andronache et al., 2013; Di Perri et al., 2013), correlated with the degree of consciousness. A modulation of DMN connectivity has been found in healthy controls during altered states of consciousness such as sedation, anesthesia (Martuzzi et al., 2010) and deep sleep (Horovitz et al., 2009). Nevertheless, the persistence of coherent DMN connectivity in several states of unconsciousness (Martuzzi et al., 2010) suggests that other phenomena may modulate the interplay between consciousness and the DMN. Concurrently with decreased DMN connectivity, a recent study detected hyperconnectivity in deep structures of the limbic system in patients with disorders of consciousness compared to healthy controls, more marked in VS than in MCS patients (Di Perri et al., 2013). This hyperconnectivity may reflect 
the persistent engagement of residual neural activity in self-reinforcing neural loops, which may disrupt normal patterns of connectivity, including synchronized neural activity in the DMN. Therefore, hypoconnectivity might not necessarily be the global hallmark of impaired consciousness, but only one aspect of a multifaceted and more complex dysfunctional architecture of brain connectivity.

A similar pattern of disrupted DMN connectivity and enhanced limbic connectivity has also been found in temporal lobe epilepsy with complex partial seizures (Pittau et al., 2012b), where transitory DMN deactivation has been reported during interictal and ictal epileptic activity. In focal epilepsy, some studies have investigated the functional connectivity of the epileptic focus, and found that patients with the most lateralized connectivity abnormalities have the best outcome after epilepsy surgery (Negishi et al., 2011). The connectivity of the epileptic focus was found to be increased between the focus and contiguous regions, decreased between the focus and remote regions and, most importantly, independent of the presence of IEDs (Luo et al., 2014). A further development in this field has been the proposal of an intrinsic connectivity contrast (ICC), "a voxel-based network measure of degree that reflects the number of connections to each voxel". A map showing the voxelwise difference between interhemispheric and intrahemisperic ICC significantly correlated with the seizure onset zone measured by icEEG (Lee et al., 2014).

In generalized childhood epilepsies recent studies have shown disrupted DMN connectivity even during interictal phases (e.g. Luo et al., 2011). Whereas most studies postulate a secondary DMN inhibition during generalized seizures, one study showed, by dynamic causal modeling, a hierarchic role of the precuneus (Vaudano et al., 2009).

To better understand the significance of RSNs disclosed by BOLD oscillations, some studies have focused on the relationships between the oscillations and EEG rhythms. Apart from the thalamic activation associated with alpha power increases, most studies did not reveal cortical correlates of scalp EEG alpha oscillations. Instead, they identified brain regions that show increased activity in the absence of alpha activity (Laufs et al., 2006). However, in most studies, the BOLD variations were correlated with an EEG measure, either single or a few channels, or a derived parameter such as global field power (Mantini et al., 2007) or global field synchronization (Jann et al., 2009). The authors of one study linked the variance of EEG spectral power at each electrode to the dynamics of different RSNs using covariance mapping, and this innovative approach gave the topographic distribution of the RSN-EEG frequency relationship (Jann et al., 2010).

A completely different way of explaining rs-fMRI data by electrophysiology has recently been proposed, and it involves the use of EEG microstates. In classical studies, Lehmann showed that the topography of the scalp electric field does not change randomly and continuously over time, but remains stable for periods of $~ 80-120 \mathrm{~ms}$; these periods of quasi-stability are termed "EEG microstates" (Lehmann, 1971). Only four different microstates are consistently observed at rest (Lehmann et al., 1998). The temporal variation of each of the four microstates, after convolution with the standard HRF, can explain a different RSN obtained by rs-fMRI (Musso et al., 2010; Britz et al., 2010). The surprising relationship between such different time scales (EEG microstates $\sim 100 \mathrm{~ms}$, and rs-fMRI $\sim 10 \mathrm{~s}$ ) has been explained by wavelet-based fractal analysis, which showed, for EEG microstates, a scale-free behavior over six scales in the 256-ms to 16-s range (Van de Ville et al., 2010).

Resting-state fMRI is a promising tool for basic and clinical research. It does not require task compliance and can therefore be easily recorded even in uncooperative patients with cognitive or consciousness impairments. However, acquisition, as well, of the physiological variables that affect the BOLD signal (Birn, 2012) can be complex, as can the interpretation of the results due to the lack of an a priori hypothesis.

\section{Concluding remarks}

In the clinical setting, functional neuroimaging is currently used to map critical functional areas prior to neurosurgical interventions, but it is likely that its application will, in the near future, be extended to the evaluation of different neurological (epilepsy in particular) and psychiatric diseases. The discovery of a "default mode network" related to slow BOLD oscillations in the resting state will allow functional neuroimaging to be extended even to the most severely impaired patients, as long as they do not move their head.

This review has provided an introductory overview of the neurovascular events underlying the BOLD signal measured by current fMRI studies, along with the electrophysiological events measured by EEG. The main clinical application of simultaneous EEG-fMRI recording is in epilepsy surgery, but combining the strengths of both these methods will allow functional neuroimaging with accurate spatial and temporal resolution, thus overcoming the limitations of $\mathrm{fMRI}$ in diseases in which neurovascular coupling is impaired. After a century of strict localization approaches to brain functions based on the loss of a function with a focal lesion (which tended to overstate the fact that the function resides there), the connectionist model is now gaining renewed interest, increasingly identifying the anatomo-functional networks of cortical-subcortical brain regions. The EEG-fMRI approach will provide important insights into the effects of disease on these anatomofunctional networks involved in human brain functions.

\section{Acknowledgments}

Flavio Villani has received a grant from Paolo Zorzi's Association for Neurosciences. Anna Vaudano is funded by grants from LICE (Lega Italiana Contro 
Epilessia, Italian league against epilepsy) and the LICE Foundation.

Paolo Vitali wishes to acknowledge Prof. Guido Rodriguez, neurophysiologist and his mentor at the University of Genoa, for stimulating his great interest in cerebral anatomofunctional networks, and Prof. Pietro Morasso, Dr Francesco Frisone, Dr Giovanni Gualniera (University of Genoa), Dr Girolamo Garreffa (E. Fermi Institute, Rome), Dr Vincenzo Roma and Dr Ivo Bruni (EBNeuro, Florence), for their technical support in pioneering studies with EEG-fMRI in Genoa.

\section{References}

Allen PJ, Josephs O, Turner R (2000). A method for removing imaging artifact from continuous EEG recorded during functional MRI. Neuroimage 12:230-239.

Allen PJ, Polizzi G, Krakow K, et al (1998). Identification of EEG events in the MR scanner: the problem of pulse artifact and a method for its subtraction. Neuroimage 8:229-239.

Andronache A, Rosazza C, Sattin D, et al. (2013). Impact of functional MRI data preprocessing pipeline on defaultmode network detectability in patients with disorders of consciousness. Front Neuroinform 7:16.

Anwar MN, Bonzano L, Sebastiano DR, et al (2009). Real-time artifact filtering in continuous VEPs/fMRI recording. J Neurosci Methods 184: 213-223.

Beckmann CF, Smith SM (2005). Tensorial extensions of independent component analysis for multisubject FMRI analysis. Neuroimage 25:294-311.

Beisteiner R, Robinson S, Wurnig M, et al (2011). Clinical fMRI: evidence for a 7T benefit over 3T. Neuroimage 57:10151021.

Benar CD, Gross DW, Wang Y, et al (2002). The BOLD response to interictal epileptiform discharges. Neuroimage 17:1182-1192.

Benuzzi F, Mirandola L, Pugnaghi M, et al. (2012). Increased cortical BOLD signal anticipates generalized spike and wave discharges in adolescents and adults with idiopathic generalized epilepsies. Epilepsia 53:622-630.

Benzagmout M, Gatignol P, Duffau H (2007). Resection of World Health Organization Grade II gliomas involving Broca's area: methodological and functional considerations. Neurosurgery 61:741-752.

Birn RM (2012). The role of physiological noise in resting-state functional connectivity. Neuroimage 62:864-870.

Boucousis SM, Beers CA, Cunningham CJ, et al (2012). Feasibility of an intracranial EEG-fMRI protocol at 3 T: risk assessment and image quality. Neuroimage 63: 12371248.

Britz J, Van de Ville D, Michel CM (2010). BOLD correlates of EEG topography reveal rapid resting-state network dynamics. Neuroimage 52:1162-1170.

Budde J, Shajan G, Zaitsev M, et al. (2014). Functional MRI in human subjects with gradient-echo and spin-echo EPI at 9.4 T. Magn Reson Med 71:209-218.

Caballero-Gaudes C, Van de Ville D, Grouiller F, et al (2013). Mapping interictal epileptic discharges using mutual information between concurrent EEG and fMRI. Neuroimage 68:248-262.

Cannestra AF, Pouratian N, Bookheimer SH, et al (2001). Temporal spatial differences observed by functional MRI and human intraoperative optical imaging. Cereb Cortex 11:773-782.

Castellazzi G, Palesi F, Casali S, et al (2014) A comprehensive assessment of resting state networks: bidirectional modification of functional integrity in cerebro-cerebellar networks in dementia. Front Neurosci 8:223.

Chang C, Thomason ME, Glover GH (2008). Mapping and correction of vascular hemodynamic latency in the BOLD signal. Neuroimage 43:90-102.

Chaudhary UJ, Duncan JS, Lemieux L (2013). Mapping hemodynamic correlates of seizures using fMRI: a review. Hum Brain Mapp 34:447-466.

Cruse D, Chennu S, Fernández-Espejo D et al (2012). Detecting awareness in the vegetative state: electroencephalographic evidence for attempted movements to command. PLoS One 7:e49933.

Cunningham CB, Goodyear BG, Badawy R, et al (2012). Intracranial EEG-fMRI analysis of focal epileptiform discharges in humans. Epilepsia 53:1636-1648.

Dai Z, Yan C, Wang Z, et al (2012). Discriminative analysis of early Alzheimer's disease using multi-modal imaging and multi-level characterization with multi-classifier (M3). Neuroimage 59:2187-2195.

Damoiseaux JS, Praterb KE, Miller BL, et al (2012). Functional connectivity tracks clinical deterioration in Alzheimer's disease. Neurobiol Aging 33:828.e19-828.e30

Daunizeau J, Grova C, Marrelec G, et al (2007). Symmetrical event-related EEG/fMRI information fusion in a variational Bayesian framework. Neuroimage 36:69-87.

Di Perri C, Bastianello S, Bartsch AJ, et al (2013). Limbic hyperconnectivity in the vegetative state. Neurology $81: 1417$ 1424.

Diukova A, Ware J, Smith JE, et al (2012). Separating neural and vascular effects of caffeine using simultaneous EEGFMRI: differential effects of caffeine on cognitive and sensorimotor brain responses. Neuroimage 62:239-249.

Fahoum F, Lopes R, Pittau F, et al (2012). Widespread epileptic networks in focal epilepsies: EEG-fMRI study. Epilepsia 53:1618-1627.

Fujiwara N, Sakatani K, Katayama Y, et al (2004). Evoked-cerebral blood oxygenation changes in false-negative activations in BOLD contrast functional MRI of patients with brain tumors. Neuroimage 21:1464-1471.

Gale SD, Pearson CM (2012). Neuroimaging predictors of stroke outcome: implications for neurorehabilitation. NeuroRehabilitation 31:331-344.

Glover GH (1999). Deconvolution of impulse response in eventrelated BOLD fMRI. Neuroimage 9:416-429.

Greicius MD, Srivastava G, Reiss AL, et al (2004). Defaultmode network activity distinguishes Alzheimer's disease from healthy aging: evidence from functional MRI. Proc Natl Acad Sci U S A 101:4637-4642.

Grouiller F, Thornton RC, Groening K, et al (2011). With or without spikes: localization of focal epileptic activity by simultaneous electroencephalography and functional magnetic resonance imaging. Brain 134:2867-2886.

Horovitz SG, Braun AR, Carr WS, et al (2009). Decoupling of the brain's default mode network during deep sleep. Proc Natl Acad Sci U S A 106:11376-11381.

Ives JR, Warach S, Schmitt F, et al (1993). Monitoring the patient's EEG during echo planar MRI. Electroencephalogr Clin Neurophysiol 87:417-420.

Jacobs J, Levan P, Moeller F, et al (2009). Hemodynamic changes preceding the interictal EEG spike in patients with focal epilepsy investigated using simultaneous EEG-fMRI. Neuroimage 45:1220-1231.

Jacobs J, Stich J, Zahneisen B, et al (2014). Fast fMRI provides high statistical power in the analysis of epileptic networks. Neuroimage 88:282-294.

Jann K, Dierks T, Boesch C, et al (2009). BOLD correlates of 
EEG alpha phase-locking and the fMRI default mode network. Neuroimage 45:903-916.

Jann K, Kottlow M, Dierks T, et al (2010). Topographic electrophysiological signatures of FMRI resting state networks. PLoS One 5:e12945.

Jorge J, Grouiller F, Ipek Ö, et al (2015). Simultaneous EEGfMRI at ultra-high field: artifact prevention and safety assessment. Neuroimage 105:132-144.

Kilner JM, Mattout J, Henson R, et al. (2005). Hemodynamic correlates of EEG: a heuristic. Neuroimage 8:280-286.

Krishnan R, Raabe A, Hattingen E, et al (2004). Functional magnetic resonance imaging-integrated neuronavigation: correlation between lesion-to-motor cortex distance and outcome. Neurosurgery 55: 904-914; discussion 914-915.

Kwong KK, Belliveau JW, Chesler DA, et al (1992). Dynamic magnetic resonance imaging of human brain activity during primary sensory stimulation. Proc Natl Acad Sci U S A 89:5675-5679.

Laufs $\mathrm{H}$, Holt JL, Elfont R, et al (2006). Where the BOLD signal goes when alpha EEG leaves. Neuroimage 31:1408-1418.

Lee HW, Arora J, Papademetris X, et al (2014). Altered functional connectivity in seizure onset zones revealed by fMRI intrinsic connectivity. Neurology 83:2269-2277.

Lehmann D (1971). Multichannel topography of human alpha EEG fields. Electroencephalogr Clin Neurophysiol 31:439449.

Lehmann D, Strik WK, Henggeler B, et al. (1998). Brain electric microstates and momentary conscious mind states as building blocks of spontaneous thinking: I. Visual imagery and abstract thoughts. Int J Psychophysiol 29:1-11.

Lemieux L, Laufs H, Carmichael D, et al (2008). Noncanonical spike-related BOLD responses in focal epilepsy. Hum Brain Mapp 29: 329-345.

Logothetis NK, Pauls J, Augath M, et al (2001). Neurophysiological investigation of the basis of the fMRI signal. Nature 412: 150-157.

Luo C, An D, Yao D, et al (2014). Patient-specific connectivity pattern of epileptic network in frontal lobe epilepsy. Neuroimage Clin 4: 668-675.

Luo C, Li Q, Lai Y, et al (2011). Altered functional connectivity in default mode network in absence epilepsy: a resting-state fMRI study. Hum Brain Mapp 32:438-449.

Ma L, Wang B, Chen X, et al (2007). Detecting functional connectivity in the resting brain: a comparison between ICA and CCA. Magn Reson Imaging 25:47-56.

Mantini D, Perrucci MG, Del Gratta C, et al (2007). Electrophysiological signatures of resting state networks in the human brain. Proc Natl Acad Sci USA 104:13170-13175

Martuzzi R, Ramani R, Qiu M, et al (2010) Functional connectivity and alterations in baseline brain state in humans. Neuroimage 49:823-834.

Meletti S, Vaudano AE, Tassi L, et al (2014). Intracranial timefrequency correlates of seizure-related negative BOLD response in the sensory-motor network. Clin Neurophysiol Aug 27 doi: 10.1016/j.clinph.2014.07.030.

Michel CM, Murray MM (2012). Towards the utilization of EEG as a brain imaging tool. Neuroimage 61:371-385.

Moeller F, Siebner HR, Wolff S, et al (2008). Changes in activity of striato-thalamo-cortical network precede generalized spike wave discharges. Neuroimage 39:1839-1849.

Monti MM, Vanhaudenhuyse A, Coleman MR, et al (2010). Willful modulation of brain activity in disorders of consciousness. N Engl J Med 362:579-589.

Morgan VL, Li Y, Abou-Khalil B, et al (2008). Development of 2dTCA for the detection of irregular, transient BOLD activity. Hum Brain Mapp 29:57-69.

Musso F, Brinkmeyer J, Mobascher A, et al. (2010).
Spontaneous brain activity and EEG microstates. A novel EEG/fMRI analysis approach to explore resting-state networks. Neuroimage 52:1149-1161.

Nagai M, Hoshide S, Kario K (2010). The insular cortex and cardiovascular system: a new insight into the brain-heart axis. J Am Soc Hypertens 4:174-182.

Negishi M, Martuzzi R, Novotny EJ, et al (2011). Functional MRI connectivity as a predictor of the surgical outcome of epilepsy. Epilepsia 52:1733-1740.

Ogawa S, Tank DW, Menon R, et al (1992). Intrinsic signa changes accompanying sensory stimulation: functiona brain mapping with magnetic resonance imaging. Proc Natl Acad Sci USA 89:5951-5955.

Pedreira C, Vaudano AE, Thornton RC, et al (2014). Classification of EEG abnormalities in partial epilepsy with simultaneous EEG-fMRI recordings. Neuroimage 99:461-476.

Petrella JR, Sheldon FC, Prince SE, et al (2011). Default mode network connectivity in stable vs progressive mild cognitive impairment. Neurology 76:511-517.

Pittau F, Dubeau F, Gotman J (2012a). Contribution of EEG/fMRI to the definition of the epileptic focus. Neurology 78:14791487.

Pittau F, Grova C, Moeller F, et al (2012b). Patterns of altered functional connectivity in mesial temporal lobe epilepsy. Epilepsia 53:1013-1023.

Power JD, Barnes KA, Snyder AZ, et al (2012). Spurious but systematic correlations in functional connectivity MRI networks arise from subject motion. Neuroimage 59:2142-2154.

Raichle ME, MacLeod AM, Snyder AZ, et al (2001). A default mode of brain function. Proc Natl Acad Sci U S A 98:676682.

Raoult H, Petr J, Bannier E, et al (2011). Arterial spin labeling for motor activation mapping at 3T with a 32-channel coil: reproducibility and spatial accuracy in comparison with BOLD fMRI. Neuroimage 58:157-167.

Rosazza C, Ghielmetti F, Minati L et al. (2013). Preoperative language lateralization in temporal lobe epilepsy (TLE) predicts peri-ictal, pre- and post-operative language performance: an fMRI study. Neuroimage Clin 3:73-83.

Rosazza C, Minati L, Ghielmetti F, et al (2012). Functional connectivity during resting-state functional MR imaging: study of the correspondence between independent component analysis and region-of-interest-based methods. AJNR Am J Neuroradiol 33:180-187.

Rossini PM, Altamura C, Ferretti A, et al (2004). Does cerebrovascular disease affect the coupling between neurona activity and local haemodynamics? Brain 127:99-110.

Ruggieri A, Vaudano AE, Benuzzi F, et al (2015). Mapping (and modeling) physiological movements during EEG-fMRI recordings: the added value of the video acquired simultaneously. J Neurosci Methods 239:223-37

Salek-Haddadi A, Diehl B, Hamandi K, et al (2006). Hemodynamic correlates of epileptiform discharges: an EEG-fMRI study of 63 patients with focal epilepsy. Brain Res 1088:148-66.

Scheeringa R, Fries P, Petersson K, et al (2011). Neuronal dynamics underlying high- and low-frequency EEG oscillations contribute independently to the human BOLD signal. Neuron 69:572-583.

Sclocco R, Tana MG, Visani E, et al (2014). EEG-informed fMR analysis during a hand grip task: estimating the relationship between EEG rhythms and the BOLD signal. Front Human Neurosci 8:186.

Sheline YI, Raichle ME, Snyder AZ, et al (2010). Amyloid plaques disrupt resting state default mode network connectivity in cognitively normal elderly. Biol Psychiatry 67:584-587. 
Storti SF, Formaggio E, Bertoldo A, et al (2013). Modelling hemodynamic response function in epilepsy. Clin Neurophysiol 124: 2108-2118.

Tana MG, Bianchi AM, Vitali P et al (2008). Exploring interregional brain interactivity in temporal lobe epilepsy using partial correlation analysis of fMRI data. Conf Proc IEEE Eng Med Biol Soc 2008:4423-4426.

Tao JX, Ray A, Hawes-Ebersole S, et al (2005). Intracranial EEG substrates of scalp EEG interictal spikes. Epilepsia 46: 669-676.

Thornton RC, Rodionov R, Laufs $\mathrm{H}$, et al (2010). Imaging haemodynamic changes related to seizures: comparison of EEG-based general linear model, independent component analysis of fMRI and intracranial EEG. Neuroimage 53: 196-205.

Van de Ville D, Britz J, Michel CM (2010). EEG microstate sequences in healthy humans at rest reveal scale-free dynamics. Proc Natl Acad Sci U S A 107: 18179-18184.

van Houdt P, Zijlmans M (2013). Different ways to analyze EEGfMRI in focal epilepsy: does it matter? Clin Neurophysiol 124:2070-2072.

van Houdt PJ, De Munck JC, Zijlmans M, et al (2010). Comparison of analytical strategies for EEG correlated fMRI data of patients with epilepsy. Magn Reson Imaging 28: 1078-86

Vanhaudenhuyse A, Noirhomme Q, Tshibanda LJ, et al (2010). Default network connectivity reflects the level of consciousness in non-communicative brain-damaged patients. Brain 133:161-171.

Vaudano AE, Ruggieri A, Vignoli A, et al. (2014). Epilepsy-related brain networks in ring chromosome 20 syndrome: an EEG-fMRI study. Epilepsia 55:403-413.
Vaudano AE, Avanzini P, Tassi L, et al (2013). Causality within the epileptic network: An EEG-fMRI study validated by intracranial EEG. Front Neurol 4:185.

Vaudano AE, Laufs H, Kiebel SJ, et al (2009). Causal hierarchy within the thalamo-cortical network in spike and wave discharges. PLoS One 4:e6475.

Vitali P, Minati L, D'Incerti L et al (2008). Functional MRI in malformations of cortical development: activation of dysplastic tissue and functional reorganization. J Neuroimaging 18:296-305.

Vulliemoz S, Carmichael DW, Rosenkranz K, et al (2011). Simultaneous intracranial EEG and fMRI of interictal epileptic discharges in humans. Neuroimage 54:182-190.

Vulliemoz S, Lemieux L, Daunizeau J, et al (2010). The combination of EEG source imaging and EEG-correlated functional MRI to map epileptic networks. Epilepsia 51:491505.

Warbrick T, Mobascher A, Brinkmeyer J, et al (2009). Single-trial P3 amplitude and latency informed event-related fMRI models yield different BOLD response patterns to a target detection task. Neuroimage 47:1532-1544.

Watanabe M, Bartels A, Macke JH, et al (2013). Temporal jitter of the BOLD signal reveals a reliable initial dip and improved spatial resolution. Curr Biol 23:2146-2150.

Wirsich J, Bénar C, Ranjeva JP, et al (2014). Single-trial EEGinformed fMRI reveals spatial dependency of BOLD signal on early and late IC-ERP amplitudes during face recognition. Neuroimage 100:325-236.

Zhou J, Greicius MD, Gennatas ED, et al (2010). Divergent network connectivity changes in behavioural variant frontotemporal dementia and Alzheimer's disease. Brain 133:1352-1367. 